tools for the successful accreditation to ISO 15189:2012 for more than 250 tests. The Department of Pathology and Laboratory Medicine was the first laboratory in KSA to gain the A2LA ISO 15189 accreditation.

\section{DEVELOPMENT OF AN ANTIMICROBIAL STEWARDSHIP EDUCATIONAL CURRICULUM FOR NURSES: THE LEARNING NEEDS ASSESSMENT}

Nour Shamas, Elham Bukhari, Asim Al Saedi, Wesam Talal Abuznadah. Infection Prevention and Control King Abdulaziz Medical City - WR

\subsection{6/bmjoq-2019-PSF.24}

Background The Antimicrobial Stewardship Program (ASP) at King Abdulaziz Medical City - Western Region (KAMC-WR) aims to improve antimicrobial use through several interventions including education of healthcare providers. Nursing represents more than a third of the health workforce at KAMCWR. Nurses' important role in facilitating the implementation of ASP has been recognized and is supported by the literature. In developing a customized educational curriculum to reinforce the role of nursing in antimicrobial stewardship (AS), this study has been done, in collaboration with the Nursing Education Department, to assess nurses' learning needs. Although studies have examined AS knowledge, there is no empirical data regarding assessment of nurses' learning needs of AS in Saudi Arabia.

Methods A descriptive, cross-sectional survey of all levels of nursing staff using a learning needs assessment tool was implemented. The tool consisted of 13 questions aiming to assess nurses' perceived knowledge of antimicrobials' spectrum of activity, usage, indication, duration, adverse effects, antimicrobial resistance, Clostridium difficile infection, vaccination, antibiotic prophylaxis, and questions related to diagnostic modalities for infectious syndromes. A five-point Likert scale was used with responses ranging from 'satisfied' to 'dissatisfied' and 'not applicable'. Questionnaires were validated by circulating the survey to ten nurses with a varying number of years of experience, and assessing their responses. Validated questionnaires were handed out by the nurse managers of all 31 NGHA departments. Simple descriptive statistics were used for data analysis.

Results The response rate was 43\% (608 of 1411). Cardiac, oncology, and surgery departments represented most of the responses. Nurses' years of experience ranged from 0 to 32 years. The majority of nurses were SN1 and 2 (56\% and $32 \%$, respectively). Knowledge was mainly lacking in identification of patients with possible $\mathrm{C}$ difficile infection, prolonged antimicrobial infusion protocols, and rapid diagnostic test sampling techniques. 'Neutral' was the mode for most answers of 8 of 13 questions, and the rest were 'moderately satisfied'. The 'not applicable' category was chosen by $2-5 \%$ of responders depending on the question. This indicates that many nurses are unsure of their need for training, and that some nurses do not even identify their crucial role in AS.

Conclusion In this study, nurses' learning needs of AS were assessed. The results show that nursing staff are in need of professional development and training on AS concepts and their key role in ASPs. The results of this survey will help the ASP at KAMC-WR build a tailored educational curriculum that aims to improve overall patient care.

\section{A QUALITY IMPROVEMENT PROJECT ON PATIENT SAFETY EDUCATION IN THE OUTPATIENT DEPARTMENT (OPD)}

Mohammad Faisal Salapuddin, Graceslick Atienza, Sitti Fatima Isnani, Mwanaid Binali. Outpatients Department, Prince Mohammed Bin Abdulaziz Hospital - Ministry of National Guard Health Affairs

\subsection{6/bmjoq-2019-PSF.25}

Background It is an ethical responsibility of registered nurses to provide health education to their patients as part of their nursing care. However, in reality, it is often not carried out because of several reasons. As a result, a quality improvement project was done in the OPD PMBAH-MNGHA. A quality team was created, which identified the absolute lack of patient education as evidenced by a survey on nurses' documentation from 1-15 February 2017. The survey showed that among 30 patients, zero patient education was provided by nurses during the period. Furthermore, a fishbone analysis showed two primary reasons for the lack of patient education: (1) unavailability of a standard patient education tool or guidelines for nurses; and (2) inadequate training support on the use of the hospital's intranet resources. Therefore, the objective of the project is to increase patient education in outpatients by at least $50 \%$ in 12 months.

Methods A PDSA (plan-do-study-act) cycle was used as a framework for developing, testing, and implementing changes for improvement. The team created a standard patient safety education guidelines tool, which contains seven topics about patient safety: medication safety, hand hygiene, fall prevention, proper patient identification, pain score tool, pressure ulcer prevention, and general control of infection. Furthermore, TeamStepps principles and strategies were used during the implementation. The outcome measure of the project was the total number of documented cases of patient education while process measures were staff documentation of patient education, staff training support, and use of patient education tool. A small test of change was done from 26-30 March 2017 prior to implementation. A simple random sampling method was used for data collection.

Results A small test of change gained a result of $80 \%$ compliance rate. From April to December 2017 compliance was $71 \%$ and in 2018 compliance was $84 \%$. The hospital's outpatient department has gone from zero patient education in February 2017 to a compliance rate of $84 \%$ in 2018 using the patient education guidelines tool as evidenced by nursing documentation.

Conclusion The project has established a quality improvement as part of ensuring the delivery of quality patient care to our clients and their families. Finally, registered nurses can perform their function as health educator to patients and their families as part of nursing care through the use of the patient safety education guidelines tool. The project can be further improved with observational study of actual patient education provided and patients' feedback. 